AIAA-2000-1378

\title{
MULTIDISCIPLINARY DESIGN TOOLS FOR AFFORDABILITY
}

\author{
Max Blair and Alicia Hartong \\ Air Force Research Laboratory \\ Air Vehicles Directorate
}

\begin{abstract}
A proven general purpose design modeling environment has been adapted to address affordability issues at the design synthesis level with the integration of Geometric Modeling and Activity-Based Cost Modeling.

Two factors make this work innovative. First, we are using an advanced design modeling environment with dependency tracking, demand-driven calculations and run-time object creation. Secondly, we explore ways this computer software innovation can be used to tightly integrate geometric modeling with activity-based cost modeling.
\end{abstract}

The example focuses on the synthesis of a hot structures solution for a high speed lifting surface.

\section{INTRODUCTION}

This paper is motivated by the AFRL Air Vehicles Directorate Vision: Develop affordable technologies for Air Force flight vehicles. Our customer values this developmental work in terms of risk reduction. Achieving this vision requires as much planning, packaging and selling as the technology development itself. The result of this preparatory work is a requirements document.

In the largest sense, customer requirements generation is a cyclical process which results in a series of increasingly detailed documents which prescribe what is to be done and how we know it is done. A requirements document is the result of much planning and results in significant testing. The product from one activity cycle becomes the requirements for the next, resulting in a higher Technology Readiness Level (TRL). Technology planners need a practical and scalable approach for inserting cost modeling into their planning process.

Adaptive Modeling Language is a trademark of TechnoSoft Inc. All other company and product names are trademarks or registered trademarks of their respective owners

This material is a work of the U.S. Government and is not subject to copyright protection in the United States.
Affordability is a significant concern to the customer getting the expected performance at the lowest cost. This concern is addressed at each TRL through a requirements document. With fiscal austerity, every technology development starts and ends with affordability. In this paper, we explore how cost aspects of affordability work in a conceptual design context and result in requirements for a technology-driven concept.

Technology integration requires us to work with a complex system in which everything depends on everything else. At first, the planner simply addresses many anticipated integration issues with insight based on experience [1]. However, technology development has become costly and risky. An affordability-conscious technology integrator works to maximize knowledge before committing to expensive developments. In doing so, there must be a transition from personal insight to a detailed model.

The cost modeling approach presented here will be part of a larger integrated process. This is achieved in part by bringing cost and geometry together into a single modeling environment. This cost model is useful to the extent that customer confidence is increased. Customer confidence is increased by decomposing the cost of activities and materials to the part level and results in a simple assembly process model. This first step toward accountability at the conceptual level increases knowledge before committing to expensive developments. This is made practical with the rapid modeling tools presented here.

Some technology developments do not raise significant integration issues. For instance, the development of tire technology can be somewhat independent of much of the vehicle system. On the other hand, materials, structures and manufacturing technologies can result in vehicle configuration changes. Where significant new technology integration issues result in comprehensive design considerations, simple weight-based parametric cost models are just plain wrong - resulting in curses 


\section{Blair and A. Hartong \\ Multidisciplinary Design Tools for Affordability}

and costly redesigns during the development stage. This is a serious problem for technology planners which may be partially solved by the cost synthesis technology presented here. This paper proposes an approach to reducing cost risk with rapid modeling using high fidelity data in early stages of configuration integration.

Affordability can be designed into a technology development program. Technology development for the warfighter usually starts with existing requirements based on current or projected capability. An affordability perspective adds value by quantifying cost/performance technology trades which are relevant to the warfighter. Subsequently, the customer can select the technologies which work for them. It follows that accurate modeling of these cost/performance trades increases awareness in the planning stage and will result in significant cost savings during the development stage.

This paper presents a number of software innovations collected into a single project called CAPTURE (Cost Activity Process design Tool in a Unified Rapid modeling Environment).

\section{THE ADAPTIVE MODELING LANGUAGE}

CAPTURE is based on the Adaptive Modeling Language (AML). AML has evolved from an in-house (Materials Directorate of the Air Force Research Laboratory) feature-based design project to a commercial product in use by industries ranging from automotive, e.g., Ford Motor and Volvo; to aerospace, e.g., Lockheed-Martin, and Boeing; and power generation, e.g., Zurn Balke-Durr and Siemens. AML supports a multidisciplinary environment for interactive product-process design.

While AML has been used to capture a number of proprietary and published design products and processes, the emphasis in this effort is to develop and integrate cost modeling aspects with geometric modeling. Here, the term integrate emphasizes the tight relationship between the geometric model and the cost model.

The Air Vehicles Directorate has a mission to develop new technology, including technology where there is more intuition than hard data. Where untested technology is planned and developments are prioritized, it is important that planners and developers have the capability to rapidly synthesize a new cost model. This was accomplished in CAPTURE by formulating AML-based objects which are composed of discrete activities and materials. At this decomposed level, planners can intelligently extrapolate from past experiences. Data for this model will be available from the VECAST [2] tool development or may come from any one of a number of other sources. This link has not yet been achieved and may require a larger effort than in-house resources can muster.

AML is an object-oriented environment with built-in dependency-tracking and demand-driven calculations which facilitate the integration and control of all aspects of the design process. With dependency tracking, AML facilitates the control of a large number of design alternatives with a single set of driving requirements. Dependency tracking can also be used to facilitate design parameterization. With demand-driven calculations, the designer can readily control when and how design information flows.

Native AML objects cover a variety of geometric constructs, non-geometric features and forms. These objects also come with an extensive suite of methods. This environment is used by a software developer to create a process which an application designer may use. Within CAPTURE, AML is used to create a process for rendering geometry and assigning manufacturing and cost intent. Subsequently, these intentions can be transformed into requirements by technology development planners. AML provides the single open-access environment which makes it practical to model a very complex process (i.e. air vehicle design integration) with a single suite of objects and methods.

References [3], [4], [5], [6] and [7] are provided here for additional background information where AML has been successfully applied to address design analysis.

\section{BACKGROUND}

In reference [3], a demonstration project was assembled in which a commercial geometric computer aided design code was used to parametrically control the geometry of the airframe outer surface and major substructures. Data was extracted with a series of configuration-dependent instructions and an aeroelastic optimization problem was accomplished for the set of parts. The process was practical for the purpose of resizing configuration and structural geometry. It was not a good environment for configuration synthesis.

In reference [4], the AML architecture was used to retain and share data with two conceptual design codes and the model in reference [3]. Again, this design process was perhaps useful for resizing a design concept. Since the participating codes were developed independently, the process had elements of redundancy and inconsistency. Also the process did not facilitate configuration synthesis. 


\section{Blair and A. Hartong \\ Multidisciplinary Design Tools for Affordability}

In reference [5], the AML architecture was used except here its unique capabilities were utilized to create paths of data feed forward and feed back for a wing, addressing preliminary weight and cost in a conceptual design study. The process facilitates structural design synthesis.

Again, in reference [6], the AML architecture was used to integrate an innovative structural concept into a vehicle design concept. The emphasis here was to demonstrate how design synthesis is facilitated by the design modeling environment. This collaborative effort was shared by AFRL, Purdue University and TechnoSoft Inc.

In reference [7], a list of functional requirements for an Aircraft Technology Assessment System (ATAS) were described. These requirements are presented in two parts. The first part describes the software requirements and the second part describes the requirements for an airframe assessment. The work presented in this paper supports the ATAS requirements.

When developing applications with dependency-tracking and demand-driven features, one needs to be aware of computational consequences. During process model development, it is important to determine which tasks should be dominated by dependency management and which tasks should be isolated for raw computational speed.

At a high level (integration), dependency-tracking and demand-driven features significantly facilitate design process development. The developer does not spend time developing a formidable flow diagram. By necessity (due to complexity) the object-code syntax is selfdocumenting.

At a low level (number crunching), these same features impose a computational penalty. Therefore, one would never want to put dependency tracking in the middle of a large matrix operation where computational speed of critically important.

\section{AFFORDABILITY PERSPECTIVES}

Affordability has become a subject of importance to our USAF customer. As this mantra filters down to the research community, the reaction has varied.

One approach is put forth by James Gregory Associates [1]. Here a technology planning team is assembled, customer requirements are identified, requirements are transformed into engineering metrics and probabilistic risk functions are assigned for a number of technology solutions. This relatively rapid process produces a rank- ing of technology candidates according to customer satisfaction with an emphasis on affordability. The capability presented in this paper complements this S\&T approach with significant details toward understanding technology integration issues.

In the context of military air vehicle development, the concept of cost takes on many meanings. One cost metric is the price paid for a production run. The motivation for this may arise from a government accounting office. On the other hand, the cost for a single vehicle is not really known and there really is no contractual requirement to calculate this figure. If one insisted, the best figure one will find is the simple average of the price for the production run divided by the number of vehicles. The actual cost of each vehicle can vary tremendously with changing work force, market forces (raw material), environmental (weather and natural disasters). The projected cost of a single prototype vehicle is even more difficult to develop.

When we address cost at the early stages of technology development we are not interested in absolute cost. We are interested in comparing costs and cost consequences. There are many ways to account for cost at the conceptual level. There are oversimplified weight-based parametric cost models based on historical regression. These are useful for projecting cost with old technology - not useful where new technology is integrated. It does not answer any integration issues - does not help us pick technologies based on the cost consequences. Cost is one of those areas where "the devil is in the details".

An alternative to weight-based parametrics is activitybased cost (ABC) models. These models are readily developed with currently available software tools as will be shown in this paper. We expect they can be usefully formulated in terms of confidence intervals for identifying cost risk. $\mathrm{ABC}$ models decompose the cost down to whatever level of detail is required to make a judgement. However, decomposing the cost in terms of materials, labor, assembly, outsourcing, capital investment and any other overhead is not the whole story. This capability has to be put into the hands of a single designer who is making rapid decisions (which have a strong influence on the cost whether he is aware of it or not).

While this paper is about designing with cost, ultimately, we have to consider not just the process but the data which goes into the $\mathrm{ABC}$ model. We do not address that aspect of cost modeling and it is considered a topic for further development. 


\section{Blair and A. Hartong \\ Multidisciplinary Design Tools for Affordability}

\section{CAPTURE}

CAPTURE will serve as an activity-based cost approach which engineers can use to rapidly synthesize an integrated geometry and cost model at the early stages of design. CAPTURE presently emphasizes structural and manufacturing aspects. Currently, these are two separate activities which will mutually benefit in far term plans.

An example where the CAPTURE cost model will be useful in the Air Vehicles Directorate arises from the current push for the development of new technology for affordable hypersonic vehicle concepts. Clearly, production data is lacking. Yet the Air Vehicles Directorate has a requirement to identify, prioritize and develop these technologies. One of the primary metrics for prioritization is cost. Consider active cooling concepts which involve the use of ceramics, hot structures and cooling channels for which we have little or no data. However, it is reasonable to expect we can develop affordability metrics at an early stage if we look at only materials and activities. We can gather this data from samples and extrapolate to a manufacturing scenario. By decomposing the data - we can target the cost and can generate a prioritized technology development strategy to reduce the cost.

With both cost and geometry objects written in AML, there is no software barrier to impede the flow of data. In fact, both the cost and geometry could not be more integrated since they are merged into the same object and object structure with automated dependency tracking. From the end-user's perspective, this means that both cost and geometry changes can be made with the same system (not separate CAD and cost programs). Furthermore, changes in the geometry are immediately reflected in the cost and if cost is an independent parametric, changes in cost are immediately reflected in the geometry.

A design process is measured by how long it takes to develop a design, how many designers are being paid (include overhead), the fidelity of the design proposal, and a probabilistic measure of risk. Automated dependency tracking will prove to reduce design time and a single system which runs both geometry and cost tends to reduce the number of designers. These savings can be transformed into increased data fidelity for a few designs or into increasing the number of designs at a lower fidelity.

\section{METHODOLOGY}

Figure (1) shows an overview of the CAPTURE methodology. It can be broken down into three primary tasks: Component Creation, Subassembly Creation, and PostProcessing. The only prerequisite for implementing activity-based cost (ABC) in CAPTURE is geometry. There are currently two options in generating the geometry needed for the ABC model. The first option is to utilize the provided geometry sketcher, thus simultaneously create both geometry and cost. This sketcher creates a conceptual wing and substructure which can be used by the cost model. This sketcher was reported in reference [5].

A second option involves two distinct processes. A geometric modeling expert completes the job of synthesizing a configuration in a CAD system of his choice, and subsequently exports the model in IGES format to be used by the cost expert. This cost expert utilizes the ABC model process in CAPTURE to develop the cost model by stepping through the three primary tasks.

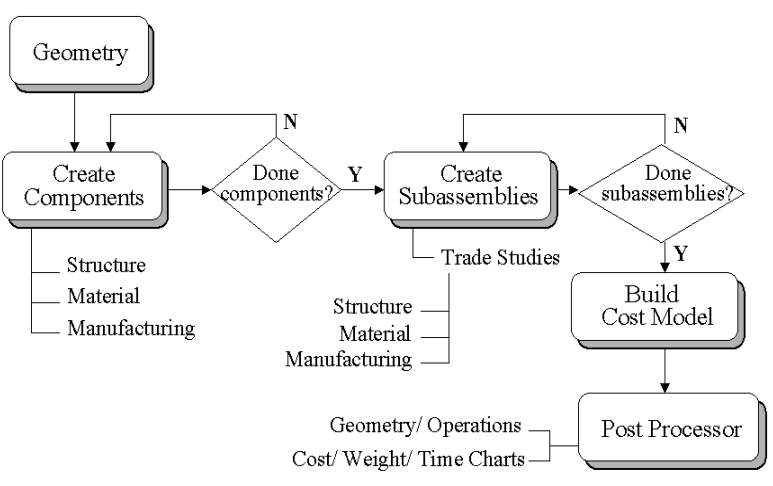

Figure (1) Methodology Overview

\section{TASK 1: COMPONENT CREATION}

Once the geometry is created/imported, the costing module can be implemented. The first task is to create unique components. This is accomplished by interactively selecting geometric parts (mouse picks) from the display screen, and assigning a material from a material catalog, a structural type from a structures library, and a fabrication sequence from an operation catalog. Assignments can be made to individual geometry objects or to a group of geometry objects that will be identical in structural type, material and manufacturing process. 


\section{Blair and A. Hartong \\ Multidisciplinary Design Tools for Affordability}

This process is accomplished using the form depicted in Figure (2).

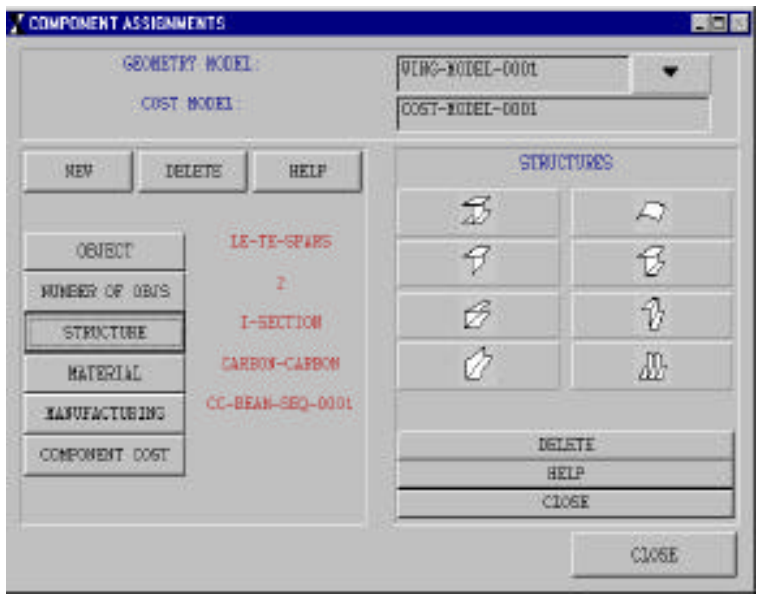

Figure (2) Component Creation Form

For each geometry selected for assignment, a component object is created and stored in a "component bin". This component object has four significant features: a pointer to the selected geometry object, a pointer to the assigned material, structural information, and a sequence of manufacturing operations. The assigned material includes both mechanical and cost data. Structural data includes geometric dimensions and area.

The assignment of manufacturing operations to any given component requires a priori knowledge of the procedure. Several manufacturing processes are available through the given Operation Catalog. These processes were gathered from technical reports, and manufacturing texts, see references [8] through [13]. The catalog is not meant to be an exhaustive, static resource, but rather a tailored, dynamic one. Operations are added to the catalog as needed, to best reflect the process activity requirements of the cost expert. One of the key features of the Operation Catalog is its ability to iteractively edit existing processes, or add new manufacturing operations and sequences. To facilitate these changes/additions a method to generate AML object code was developed. This method writes to a file all the properties and formulas that the user developed in real time. Thus, the Operation Catalog is automatically updated within the session as well as updated for permanent storage within the database.

The default times for the processing operations are estimates based on geometric characteristics such as surface-area, perimeter, length, thickness, etc. However, the cost expert has easy access to the operation's time estimates through a graphical user interface. Likewise, other properties, such as labor rate $(\$ / \mathrm{hr})$, can be changed using the Edit Operation form as shown in Figure (3).

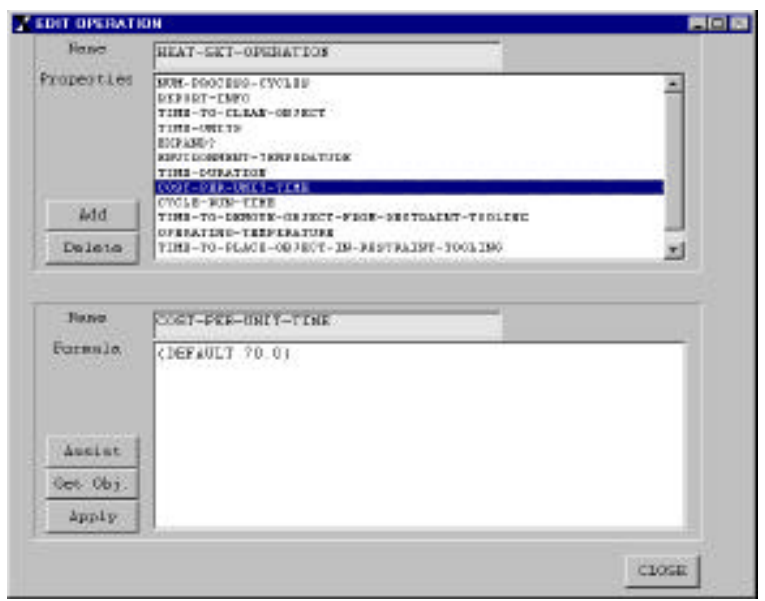

Figure (3) Edit Operation Form

In general terms, the manufacture of a component occurs by a single operation or by an ordered sequence of operations. The user can browse through the Operation Catalog by utilizing the quick view capability. This allows the user to get a top-level view of any selected operation's properties and children (operation sequences). In order to assign an operation or predefined operation sequence, the user must select it from the Operation Catalog, and then transfer it to the Operation Sequence list using the right arrow button. This notifies CAPTURE that the selected process is required to define the component's fabrication. For a multi-step manufacturing procedure the user continues to select and transfer operations to the Operation Sequence list in a user-prescribed order. The form which drives a carbon-carbon woven beam is depicted in Figure (4).

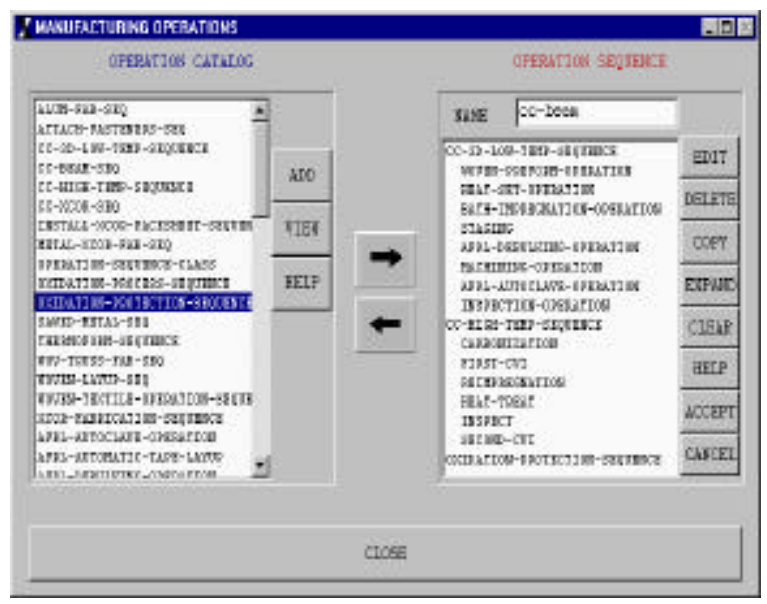

Figure (4) Operation Catalog 
All the operations in the Operations Sequence list are stored in a temporary "Operations Bin". The Operations Bin manages the processes for any copying, editing, or deleting prescribed by the user. When the user finalizes the Operations Sequence list, it is assigned to the active component(s) within the Component Bin, and deleted from the Operations Bin.

\section{TASK 2: SUBASSEMBLY CREATION}

Once the components have been created, the user begins the second task of creating subassemblies. Two-part in nature, this task requires the user to group any number of components and/or subassemblies together, and apply manufacturing assembly techniques to join the components into a unified assemblage.

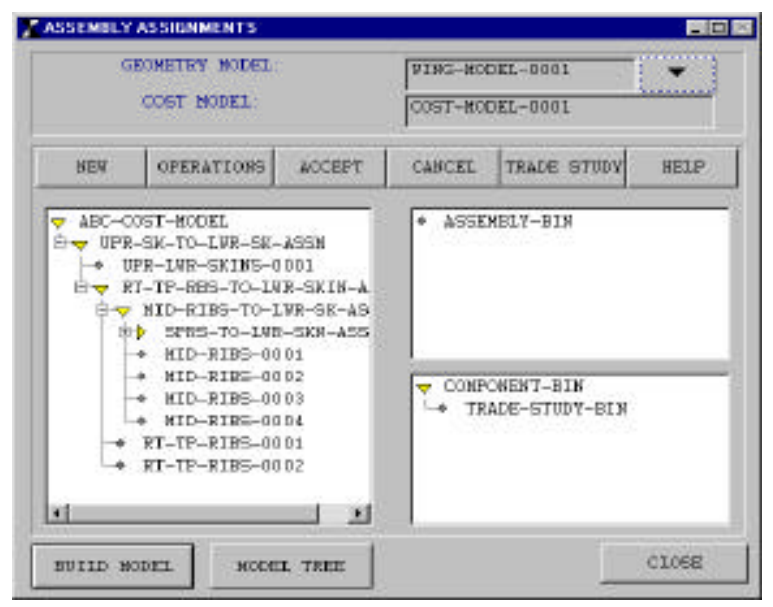

Figure (5) Subassembly Creation Main Form

A bottoms-up method is used to create a subassembly. First, the user checks out desired components from the Component Bin and transfers them to a user-named subassembly object. Next, the user utilizes the Operation Catalog to assign the manufacturing assembly operation, such as bonding, mechanical fastening, or welding to the subassembly. Then, the user "accepts" his newly created subassembly, and it is transferred to the Assembly Bin. In a like manner, the user continues to create subassemblies from predefined components and/or predefined subassemblies by accessing the Component and Assembly Bins.

Another task complementary to creating subassemblies is building the cost model. This requires the user to group the assigned components and subassemblies in a top-down manner. Depending on the how the root of the cost model is made, the user may be required to assign an assembly operation to the root object. Figure (5) shows the subassembly creation main form, with an assembled cost model on the left and the Assembly and Component Bins on the right.

The subassembly creation main form provides two additional capabilities. The first capability provides a means to investigate trade study scenarios; CAPTURE creates an identical copy to the component to be studied, and places both the baseline component and the copied component in the Trade Study Bin. In the trade study mode, the user can query assignment details, edit structural, material, and manufacturing definitions, and view weight, process times, total, process, and material costs for each component. Additional copies of the baseline component or the copied component can be generated to investigate further trades. Further, any object in the trade study bin can be deleted by the user. Upon exiting the trade study form, all trade study objects are transferred to the Component Bin as selectable components for subassembly and model creation. In its current development, CAPTURE allows the user to copy entire subassemblies to study different grouping scenarios, as needed for manufacturing cell formation, but does not track the necessary changes to the assembly procedures.

The second capability is a model tree viewer of component and subassembly cost, weight and process time metrics. Similar to the trade study viewing function for individual components, this provides the user a top-level view of different metrics applied to the entire cost model. In addition, AML provides the ability to save a working or complete model for later use. This feature is made available to the user through the model tree viewer as well as through the AML main menubar.

\section{TASK 3: POST-PROCESSING}

A graphical post-processor has been developed in CAPTURE to provide the user an additional means to query and view the cost model results. Here, the user visually grasps the tight integration of geometry with cost. As indicated earlier, while the combined geometry and cost models are developed interactively, they automatically maintain dependency. Changes in the geometry are immediately reflected in the cost model (when the cost is demanded).

The post-processor has three main features: a cost model flowchart, a geometry and fabrication viewer, and bar and pie charts for weight, process time, and cost metrics. The cost model flowchart exhibits the top-down associations between subassemblies and components. The subassemblies are outlined in green and the components are outlined in blue. When a user selects a component or subassembly block with the geometry option, the geometry and fabrication procedure to create it are displayed. 


\section{Blair and A. Hartong \\ Multidisciplinary Design Tools for Affordability}

When the user activates the graph option and selects a subassembly, the metric of choice, (e.g. weight, process time or cost), is graphed using a bar or pie chart. The example at the end of the paper demonstrates these capabilities and depicts the corresponding forms. Likewise, the user can graph a component's manufacturing process time and cost. The capability to graph a component's weight, material or total cost is deactivated since it is a point reference only.

\section{AML ENHANCEMENTS}

Reparenting: This feature was recently developed by TechnoSoft to address the need for moving objects from one location to another within the model tree.

Copy-Object: Another enhanced feature is a method to copy an existing instance. This method was developed in CAPTURE to facilitate trade studies and what-if scenarios. It allows the user to replicate an object's properties and associated geometry. This method is recursive in nature copying the property and geometry information of an object's children as well.

\section{EXAMPLE CASE}

DESCRIPTION: A wing structure with peak operating temperatures of $2200 \mathrm{~F}$ was selected as the test case. The wing has a root chord of $164.0 \mathrm{in}$, tip chord of 39.0 in, and a semi-span of 180.0 in. It uses a NACA-0004 airfoil section. The wing consists of sixteen components: skins(2), spars(8), and ribs(6) which are mechanically attached with SiC/C fasteners. A uniform thickness is applied to all components. A carbon-carbon material is prescribed for all the components.

In order to satisfy this top-level thermal requirement, a carbon-carbon material (C-C) with oxidation protection was utilized. The manufacture of a carbon-carbon material involves many complex processing steps, see references [8] through [11]. These include a low temperature process sequence, a high temperature process sequence, and application of an oxidation protection coating. The low temperature processing of a C-C material is similar to a resin matrix composite involving the following steps: impregnation, layup, molding, and machining. The high temperature processing includes a carbonization step, and two chemical vapor infiltration (CVI) steps separated by a reimpregnation, pyrolysis and heattreatment. The oxidation protection processing involves three different surface applications. After the initial surface preparation, a pack cementation coating is applied. Next, a multi-layer silicon-carbide ( $\mathrm{Si}-\mathrm{C}$ ) coating is applied by chemical vapor deposition (CVD). Finally, an external glaze layer overcoats the silicon-carbide layer to provide additional oxidation and moisture protection. CAPTURE accounts for these manufacturing processes by tracking the numerous activities through its Operations Catalog.

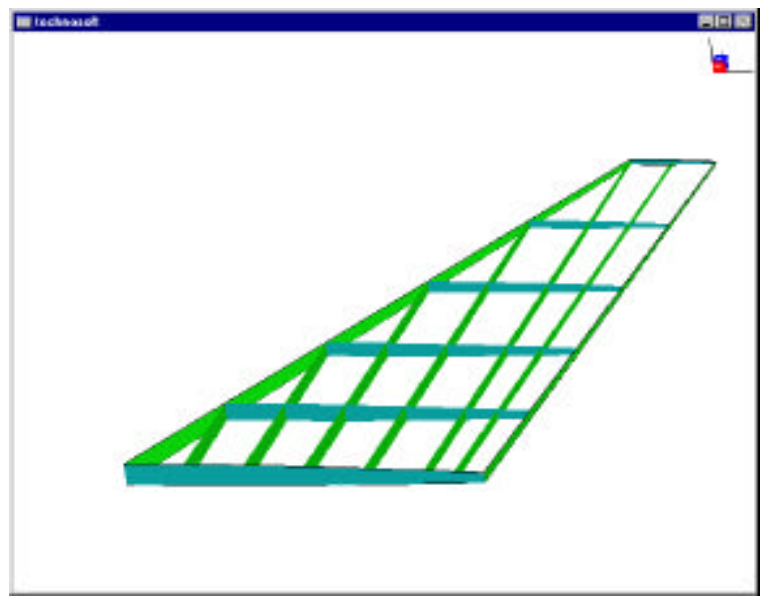

Figure (6) Wing Structure

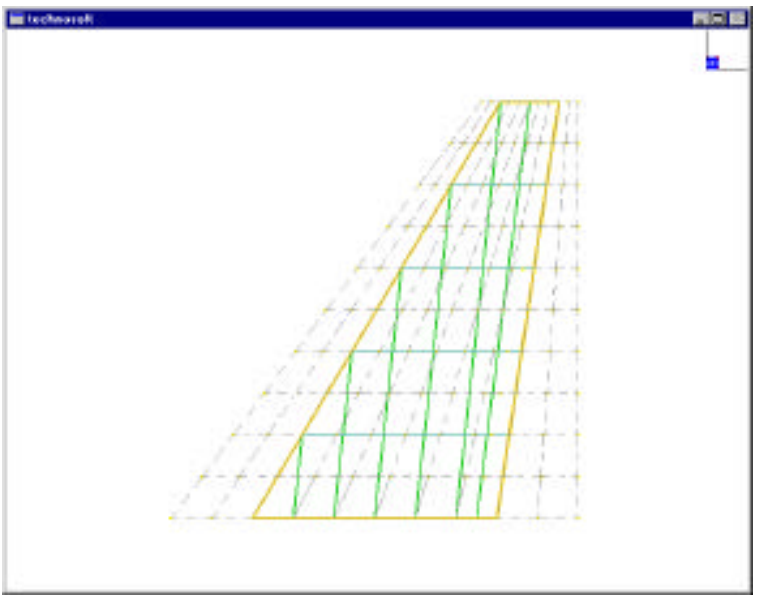

Figure (7) Substructural Layout

GEOMETRY SYNTHESIS: In Figure (6) we begin the process to generate an engineering projection of cost for undeveloped technology. Here, a wing structure is depicted. This was developed independently of the cost tool.

In order to generate the geometry in Figure (6), the surface is modeled using several geometric parameters which define the outer moldline. In addition, modeling of the wing surface entails laying out substructure, and skinning the substructures to create rib, spar, and skin surfaces. See reference [5] for additional details on the geometry creation process. The total time to create the wing structure surfaces in AML on a $233 \mathrm{MHz} P C$ was 3 minutes and 14 seconds of wall clock time. Figure (7) 
shows the generated substructure and planform. A detailed proposal for developing data for composite process modeling is presented later in this paper.

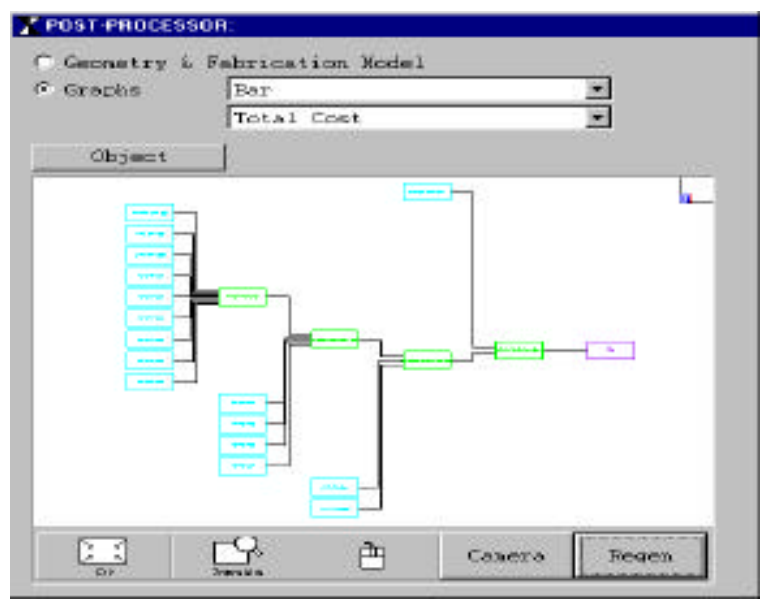

Figure (8) Cost Model Flowchart

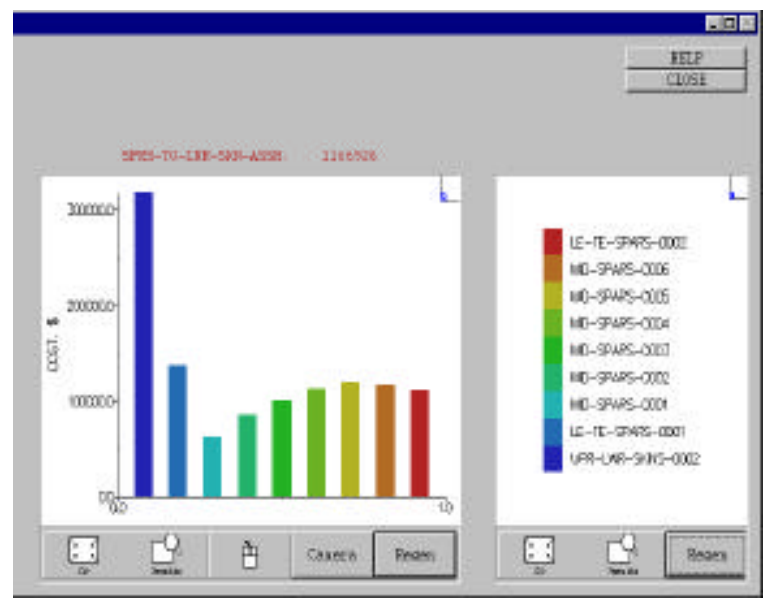

Figure (9) Subassembly Total Cost Bar Graph

COST SYNTHESIS: The cost modeling of the wing entails three operations. The first requires transforming the geometry into components having structural, material, and manufacturing characteristics. The second requires grouping the components into subassemblies having specified associations between selected components through the assignment of assembly-type manufacturing processes. The third operation requires grouping the subassemblies and/or components with corresponding assembly-type manufacturing processes into a top-down cost model. The total time to create the cost model for this example was 9 minutes and $37 \mathrm{sec}-$ onds of wall clock time. The time required to assign the sixteen components was 6 minutes and 10 seconds. The time required to assign the subassemblies and build the final cost model was 3 minutes and 20 seconds. Postprocessing time to generate the flowchart and graph the total cost for a subassembly was 7 seconds. Figures (8) and (9) are the left and right side respectively of a single post-processor form. These figures depict the cost model flowchart and the total cost bar graph for a subassembly respectively.

REDESIGN: The redesign affected three geometric parameters. The root chord was changed from 164.0 in to $220.0 \mathrm{in}$. The semi-span was changed from 180.0 to 300.0 in, and the airfoil section was changed from a NACA-0004 to NACA-4404. The time to make these changes and then re-calculate the total-cost for the same subassembly as in Figure (9) was 2 minutes and 28 seconds. The subassembly total cost bar graph for the redesign is shown in Figure (10).

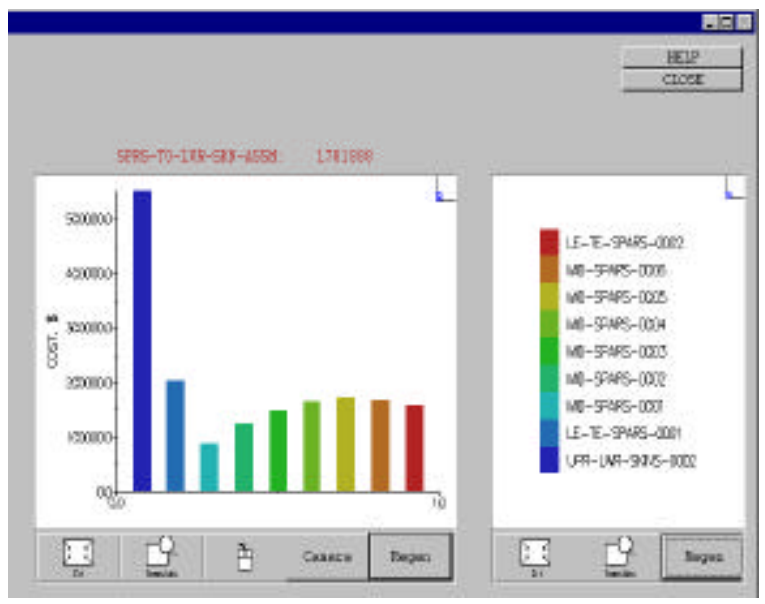

Figure (10) Redesigned Subassembly Bar Graph

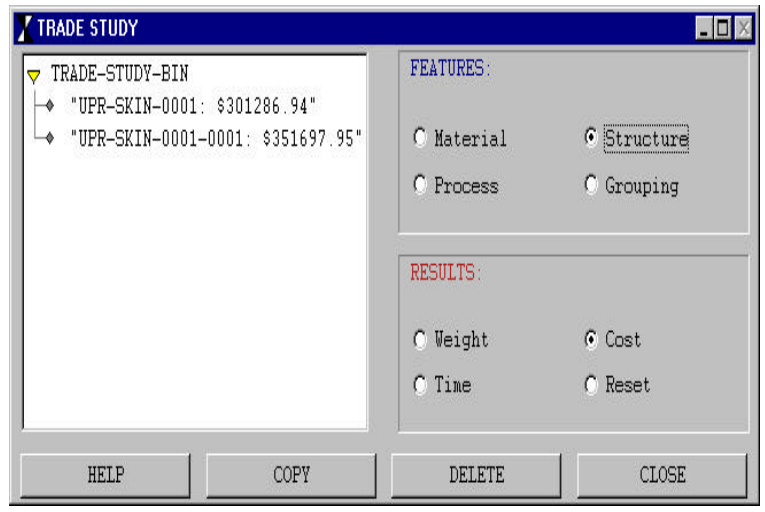

Figure (11) CC Skin vs. CC-Xcor Skin Trade Study 
TRADE STUDY: An example trade study was performed on the original test case. It consisted of swapping out the structure definition of a woven carboncarbon upper skin for a x-cor sandwich upper skin having carbon-carbon facesheets and a foam-pin core of Rohacell 31IG foam and SiC/C pins. Figure (11) shows the process cost of the initial upper skin definition; as well as the process cost of its copy with altered material and manufacturing assignments. The wall clock time to initiate these changes was 2 minutes and 12 seconds.

DISCUSSION: The costs represented are not intended as absolutes, rather they provide valuable insights into the design decision process in the form of a delta factor.

The wall clock times recorded in this test case do not reflect the time required to generate a new manufacturing process. All selected operations had been previously generated with acceptable default values. As new technologies are encountered and desired, inclusion into the Operation Catalog is necessary. The user required time is a function of the extent of calibration to an existing process definition, and the level of detail the cost expert requires in the definition of a new manufacturing process for a component and/or subassembly fabrication.

\section{LINKING ANALYSIS WITH MANUFACTURING}

Activity-based cost modeling of a composite part requires knowledge about the part layup. Here, we are proposing to address manufacturing issues at the same time we are developing analyses. This will be accomplished to the extent that stacking sequence is addressed efficiently and simultaneously with finite element analysis. The proposed process accomplishes this in several steps each of which adds additional detail.

The stiffness $\bar{Q}_{i j}$ relation between strain and stress for a single oriented lamina is drawn from Reference [14].

$$
\left[\begin{array}{c}
\sigma_{1} \\
\sigma_{2} \\
\sigma_{12}
\end{array}\right]=\left[\begin{array}{lll}
\bar{Q}_{11} & \bar{Q}_{12} & \bar{Q}_{16} \\
\bar{Q}_{12} & \bar{Q}_{11} & \bar{Q}_{26} \\
\bar{Q}_{16} & \bar{Q}_{26} & \bar{Q}_{66}
\end{array}\right]\left[\begin{array}{l}
e_{1} \\
e_{2} \\
e_{12}
\end{array}\right]
$$

Classical linear laminate analysis would have us integrate the stiffness over a finite number of layers of lamina based on the Kirchoff-Love hypothesis (a linear variation of strain through the laminate thickness) as follows.

$$
\left[\begin{array}{c}
N_{x} \\
N_{y} \\
N_{x y}
\end{array}\right]=\sum_{k=1}^{N}\left[\int_{z_{k-1}}^{z_{k}}[\bar{Q}]_{k}\left\{\left[\begin{array}{c}
e_{1}^{0} \\
e_{2}^{0} \\
e_{12}^{0}
\end{array}\right]+\left[\begin{array}{c}
\kappa_{1}^{0} \\
\kappa_{2}^{0} \\
\kappa_{12}^{0}
\end{array}\right] z\right\} d z\right]
$$

and

$$
\left[\begin{array}{c}
M_{x} \\
M_{y} \\
M_{x y}
\end{array}\right]=\sum_{k=1}^{N}\left[\int_{z_{k-1}}^{z_{k}}[\bar{Q}]_{k}\left\{\left[\begin{array}{c}
e_{1}^{0} \\
e_{2}^{0} \\
e_{12}^{0}
\end{array}\right] z+\left[\begin{array}{c}
\kappa_{1}^{0} \\
\kappa_{2}^{0} \\
\kappa_{12}^{0}
\end{array}\right] z^{2}\right\} d z\right]
$$

If the number of layers $N$ is large, this results in a large number of design variables involving the thickness, fiber orientation and material in each layer. Here we propose to reduce the number of variables.

Let us assume a laminate plate is comprised of a set of $n$ basis lamina which may have any one of $n$ orientations. At any plane, $z$, within this laminate we imagine an infinitely thin lamina with a designed stiffness $[\bar{Q}]_{A}$ formulated as some weighted combination of the stiffness from the $n$ basis lamina.

$$
[\bar{Q}]_{A}=\sum_{i=1}^{4} w_{i}[\bar{Q}]_{i}
$$

where

$$
\sum_{i=1}^{4} w_{i}=1
$$

With an infinitely thin lamina, we can continuously differentiate a variable $[\bar{Q}]_{A}$. Therefore, a laminate with thickness $t$ would have stiffness formulated as

$$
\left[\begin{array}{c}
N_{x} \\
N_{y} \\
N_{x y}
\end{array}\right]=\int_{-t / 2}^{t / 2}[\bar{Q}(z)]_{A}\left\{\left[\begin{array}{c}
e_{1}^{0} \\
e_{2}^{0} \\
e_{12}^{0}
\end{array}\right]+\left[\begin{array}{c}
\kappa_{1}^{0} \\
\kappa_{2}^{0} \\
\kappa_{12}^{0}
\end{array}\right] z\right\} d z
$$

and

$$
\left[\begin{array}{c}
M_{x} \\
M_{y} \\
M_{x y}
\end{array}\right]=\int_{-t / 2}^{t / 2}[\bar{Q}(z)]_{A}\left\{\left[\begin{array}{c}
e_{1}^{0} \\
e_{2}^{0} \\
e_{12}^{0}
\end{array}\right] z+\left[\begin{array}{c}
\kappa_{1}^{0} \\
\kappa_{2}^{0} \\
\kappa_{12}^{0}
\end{array}\right] z^{2}\right\} d z
$$

Several examples are given here in order to put this approach into context. 


\section{Blair and A. Hartong \\ Multidisciplinary Design Tools for Affordability}

If we have $n=1$ basis lamina then equations (6) and (7) would be equivalent to equations (2) and (3).

If we have $\mathrm{n}=2$ basis lamina and $w_{i}=1 / 2$ throughout the thickness, then equations (6) and (7) would approximate equations (2) and (3) for discrete ply thickness as the number $N$ of plies approaches infinity. This could be a cross-ply laminate.

Now assume we have $n=4$ basis lamina and $w_{i}=w_{i}(z)$ through the thickness as depicted in Figure (12). We enforce equation (5) at any point $\mathrm{z}$ in the thickness. Again, equations (6) and (7) would approximate equations (2) and (3) for discrete ply thickness as the number $N$ of plies approaches infinity. It remains to be shown how to reverse-engineer a discrete (i.e. a manufacturable) ply layup so equations (2) and (3) would closely approximate the designed stiffness produced using equations (6) and (7).

If we assume $w_{i}(z)$ is depicted in Figure (12), then we have 20 degrees of freedom for an element (assuming the layup is invariant with respect to $x$ and $y$ ). This is a manageable number of variables.

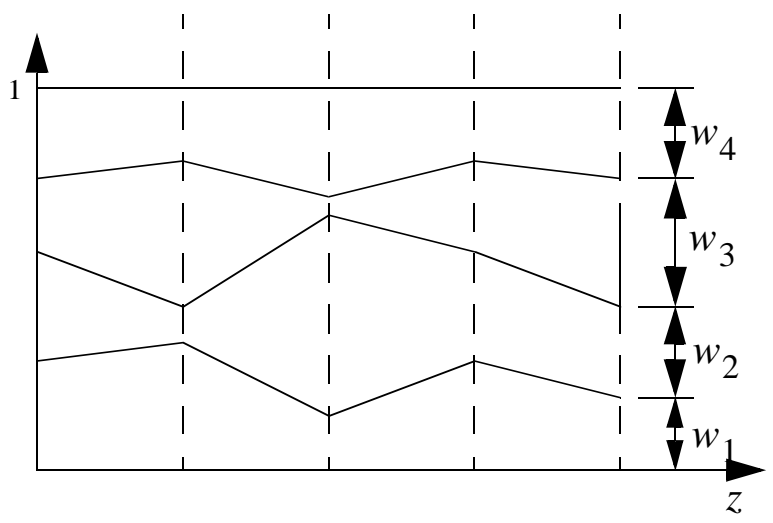

Figure (12) Redesigned Subassembly Bar Graph

Consider the design of a composite wing skin. A process is described to incrementally increase the design complexity and deliver a model which readily translates into composite process modeling information.

A design process starts with a quasi-isotropic lamina basis [0/90/土45]. Each finite element has one design variable, the laminate thickness. The vehicle structure is optimized for thickness alone.

Next, the weighting function $w_{i}(z)$ is introduced for each element, assuming uniform material through the thickness. The vehicle structure starts with the quasi- isotropic laminate design and is now optimized for thickness and weighting.

Finally, the weighting function assumes a variation throughout the thickness, perhaps as indicated in Figure (12). The vehicle structure starts with the uniform laminate design and is now optimized for thickness, weighting and distribution (with respect to $\mathrm{z}$ ). The designer may elect to accomplish this last step as an independent substructure with loads from the previous case.

If we start with the final step (with all variables free), the designer will be very uncertain the optimization converged on the minimum (e.g. weight) solution. In this incremental process, we will achieve a solution with higher confidence that we have not simply converged on a local minimum. Just as important, the final converged finite element solution tells the manufacturing designer a significant amount of information about the ply layup sequence. Finally, this will be achievable with a single incremental design model.

\section{FUTURE ACTIVITIES}

The cost modeling effort at the conceptual level must be viewed non-deterministically for a cost-risk reduction process. In order to reflect this in CAPTURE, we propose to develop a confidence-object which can be inherited along with any property-object to create a new confidence-property-object. In this way, non-deterministic quantities can be rapidly rolled up along with other deterministic quantities.

The developments presented here have been accomplished with a small investment. Meanwhile, scarce research funding requires that we temporarily put this work on hold.

In keeping with the mission of the MultiDisciplinary Technology Center, we seek avenues to incorporate the cost modeling developments presented here with other ongoing developments within the Air Vehicles Directorate.

\section{CONCLUSION}

Clearly there is a motivation for technology planners to present an accurate understanding of affordability issues before embarking on an expensive technology development program. The processes presented in this paper demonstrates an effective approach which decomposes the cost model to the part level commensurate with the technology. This is accomplished in a modeling environment which facilitates the integration of cost and 
geometry into a single activity-based cost modeling process.

\section{REFERENCES}

[1] James Gregory Associates, Inc., Affordability in Science and Technology, http://www.jamesgregory.com/

[2] Robert Quaglieri and Max Blair, "Approaches to S\&T Cost Modeling at the U.S. Air Force Research Laboratory/Vehicle Aeronautics Directorate", NATO RTO Specialists' Meeting on Design for Low Cost Operation and Support at Ottawa, Canada 21-22 October 1999

[3] Max Blair, Geetha Bharatram, Robert A. Canfield, "Designing a Blended Composite Wing and Fuselage", AIAA paper 96-3995 presented at the 6th AIAA/NASA/ ISSMO Symposium on MultiDisciplinary Analysis and Optimization 4-6 September 1996 in Bellevue WA

[4] Max Blair, Steven R. LeClair, Jeffrey V. Zweber and Adel Chemaly, "MultiDisciplinary Design for Uninhabited Air Vehicles", presented at the IEEE Sixth Workshop on Enabling Technologies: Infrastructure for Collaborative Enterprises, 18-20 June 1997 at Cambridge MA.

[5] Jeffrey V. Zweber, Max Blair, Geetha Bharatram, Hilmi Kamhawi, Alicia Hartong, "Structural and Manufacturing Analysis of a Wing Using the Adaptive Modeling Language", AIAA paper 98-1758 presented at the 39th AIAA/ASME/ASCE/AHS/ASC Structures, Structural Dynamics and Materials Conference 20-23 April 1998 in Long Beach CA.

[6] Max Blair, Stephen Hill, Terrence A. Weisshaar, Robert Taylor, "Rapid Modeling with Innovative Structural Concepts", AIAA paper 98-1755 presented at the 39th AIAA/ASME/ASCE/AHS/ASC Structures, Structural Dynamics and Materials Conference 20-23 April 1998 in Long Beach CA.

[7] Duane F. Veley, Maxwell Blair, Jeffrey Zweber, "Aircraft Technology Assessment System", AIAA paper 98-4825, presented at the 7th AIAA/NASA/ ISSMO Symposium on MultiDisciplinary Analysis and Optimization 2-4 September 1998 in St. Louis MO.

[8] Steven Hahn, et al. "Carbon-Carbon Primary Structure - Final Report” WL-TR-95-3058.

[9] "Carbon-Carbon Primary Structure - Technical Operating Report - Fabrication Plan” 4 February 1991.
[10] G. Savage, Carbon-carbon composites, Chapman \& Hall, New York 1993.

[11] J. Buckley, Carbon-carbon materials and composites, Noyes Pub., Park Ridge NJ, 1993.

[12] Northrop Corp., “Advanced Composite Cost Estimating Manual” AFFDL-TR-76-87.

[13] E. Kunkel, and T. Campbell, "Phase II Truss Reinforced Core" AFW-3211-FM-95212-1148.

[14] Robert M. Jones, Mechanics of Composite Materials, Scripta Book Co. Washington DC 1975 